\title{
Analyse économique et facteurs d'adoption de la complémentation des boeufs de traction au Mali
}

\author{
RH Bosma 1, H Dioura 2, S Bagayogo 2, K Bengaly 2 \\ 'Roghorst 363, Wageningen, Pays-Bas; ${ }^{2}$ ESPGRN, BP 186, Mali
}

Au Mali-sud, les boeufs de traction sont très sollicités dès les premières pluies alors qu'ils ont perdu $20 \%$ de leur poids vif pendant la saison sèche. Néanmoins, leur efficacité ne semble pas liée au poids vif s'ils travaillent moins de 5 heures.jour ${ }^{-1}$ (ERSP CRZ, 1990. Atelier Prod Anim et Cult Fourr, IER USAID, Bamako). Certains paysans utilisent ainsi plusieurs paires de boeufs alternativement, chacune 5 heures.jour ${ }^{-1}$, tout en reconnaissant que le nombre d'animaux nécessaires peut être diminué lorsque ceux-ci sont en bon état.

En 1992, des essais menés chez les paysans ont comparé quatre types de compléments sur des boeufs en régime de pâture journalière pendant la saison sèche en zone sub-humide : bloc Métocour, consommé en moyenne à raison de $250 \mathrm{~g}$. UBT- ${ }^{-1}$ (Unité Bétail Tropical de $250 \mathrm{~kg}$ ) $<1>$, foin de sorgho ou mil $<2>$, mélange de pailles de maïs et de Dolichos lab-lab (semé en dérobé) $<3>$, pailles hachées de sorgho enrichies $<4>$ (Bosma et Bengaly, Ann Zoot, 1996), $(<2\rangle,\langle 3\rangle,<4>: 2 \mathrm{~kg}$ complément/tête/jour).

En valeur monétaire, le gain de poids n'équilibre pas le coût de la complémentation. En incluant les heures de travail supplémentaires, la rentabilité est positive. Le complément maïs/dolique lab-lab produit le coût d'opportunité et la marge brute les plus favorables.

en F.CFA/paire de boeufs

\begin{tabular}{r}
\hline$<1\rangle$ \\
7,806 \\
5,180 \\
$-2,626$ \\
14,774 \\
12,148
\end{tabular}

Coût complément

Gain de poids

Marge brute 1

Valeur travail

Marge brute 2
La comptabilisation du fumier dont l'intérêt est exprimé par les paysans (Diabaté et al, 1994, ESPGRN, 94/03) génère la marge maximale avec une complémentation en stabulation comme le montre un essai de 1991 comparant un régime de paille enrichie en stabulation et deux lots au pâturage complémentés soit avec les blocs métocour soit avec de la paille de sorgho simple.

Le taux de rémunération (recette/ dépenses) (Crawford, 1987, Mich State Univ Intern Dev Papers, N $7 F$ ) est supérieur pour le système le moins intensif (381 vs $206 \%$ ) et le taux de rentabilité de la complémentation (bénéfice supplémentaire / charges supplémentaires) est faible (Métocour: $48 \%$; stabulation: $70 \%$ ). Des paysans adoptent pourtant la complémentation, souvent la plus coûteuse (sous- produits agroindustriels), car aux éléments comptables s'ajoutent des facteurs de décision difficilement chiffrables : complément réduisant la mortalité par sous alimentation et fidélisant les animaux (moins de pertes en brousse) ; contraintes de stockage de fourrages et charge de travail saisonnière excessive pour leur culture ; possibilités de crédits pour l'achat des sous produits.

Plusieurs stratégies doivent être offertes, les facteurs de décision variant d'un individu à l'autre.

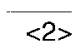

8,946

3,360

$-5,586$

12,616

7,030

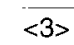

7,506

6,300

$-1,206$

14,442

13,236

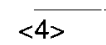

7,866

2,030

$-5,836$

15,438

9,602

Paille $\mathrm{S}$
0
6640
0
6640

Métocour
6558
9960
1925
5237

Stabulation
11640
21435
3413
13208

\author{
en F,CFA/paire de boeufs \\ Coût aliment \\ Valeur travail+ gain de poids \\ Marge 3
}

